\title{
Energy efficiency analysis of high speed triple-play services in next-generation PON deployments
}

\author{
S. Lambert ${ }^{\mathrm{a}, *}$, B. Lannoo ${ }^{\mathrm{a}}$, A. Dixit ${ }^{\mathrm{a}}$, D. Colle ${ }^{\mathrm{a}}$, M. Pickavet ${ }^{\mathrm{a}}$, J. Montalvo $^{\mathrm{b}}$, J.A. Torrijos $^{\mathrm{b}}$, P. Vetter $^{\mathrm{c}}$ \\ ${ }^{a}$ Ghent University-iMinds, Department of Information Technology, Ghent, Belgium \\ ${ }^{b}$ Telefónica I+D, Access Network Evolution, Madrid, Spain \\ ${ }^{c}$ Alcatel-Lucent Bell Labs, Fixed Networks, Murray Hill, NJ, US
}

\begin{abstract}
In this paper, the energy consumption of high speed access services up to $1 \mathrm{~Gb} / \mathrm{s}$ per customer is estimated for different passive optical network (PON) technologies. While other studies on PON power consumption typically assume a fixed split ratio, we also consider a greenfield approach, where the split ratio can be optimized for each technology, taking full advantage of its capacity and reach. The split ratio optimization takes into account Quality of Service (QoS) in terms of bandwidth availability and packet loss for triple-play services (voice, television and Internet). This paper includes an in-depth discussion of our split ratio dimensioning approach and our power consumption model for an optical access network in a major city. The obtained results show that statistical gain provided by dynamic bandwidth allocation as well as power splitting ratio optimization in PONs are key factors for achieving energy efficiency. For access rates up to $900 \mathrm{Mb} / \mathrm{s}$, XG-PON1 turns out to be the most energy efficient option. For higher access rates up to $1 \mathrm{~Gb} / \mathrm{s}$, the optimal technology depends on split ratio restrictions. If an existing optical distribution network (ODN) with split ratio 1:64 is used, XG-PON1 remains the most energy efficient technology. If higher split ratios up to 1:256 can be achieved, TWDM PON becomes the most energy efficient solution for access rates up to $1 \mathrm{~Gb} / \mathrm{s}$.
\end{abstract}

Keywords: energy efficiency, next-generation passive optical network, dynamic bandwidth allocation, network planning, high speed access

\section{Introduction}

Fiber-based passive optical networks (PONs) are currently being deployed by operators in several countries, offering much higher bandwidths than traditional copper-based access networks. Deployments of $2.5 \mathrm{~Gb} / \mathrm{s}$ capable PONs (Gigabitcapable PON or GPON) are currently the most common, while $10 \mathrm{~Gb} / \mathrm{s}$ capable PONs (next-generation PON or NG-PON) are expected in the next couple of years. In the long term, increasing bandwidth demands associated with mobile backhauling, low-latency cloud services and the convergence of residential and business access will necessitate the deployment of even faster next-generation PONs beyond $10 \mathrm{~Gb} / \mathrm{s}$, referred to as NGPON2s by the Telecommunication Standardization Sector of the International Telecommunication Union (ITU-T) and Full Service Access Network (FSAN) Group [1].

At the same time, there is a growing interest in reducing the energy consumption and the associated cost of the access network. Due to rising energy prices and the growing awareness of climate change, energy efficiency is becoming an important factor when analyzing the operational expenditures and carbon footprint of communication networks such as NG-PON(2) systems.

\footnotetext{
${ }^{*}$ Corresponding author. Tel.:+32933 14977.

Email addresses: sofie.lambert@intec.ugent.be (S. Lambert), jmg@tid.es (J. Montalvo), peter.vetter@alcatel-lucent.com (P. Vetter)
}

Estimations of energy consumption of NG-PON(2) technologies have already been reported, providing the total energy consumption per customer considering both network operator and customer premises contributions [2, 3]. Some proposals have recently shown the potential for reduction of the energy consumption in customer premises equipment (CPE), for example using a bit-interleaving protocol at the optical networking unit (ONU) in time-division multiplexing PONs [4] or a networkenhanced residential gateway approach [5]. Regarding the energy consumption associated with the network operator, energy efficiency scenarios for long-reach GPON technologies with an optimized number of central offices (COs), have been reported in [6].

Nevertheless, previous works have paid little attention to the provided services, the statistical gain of dynamic bandwidth allocation and the quality of service (QoS) achieved by each PON technology, and only considered the maximum speed capacities of both optical line terminal (OLT) and ONU. The contribution of this work is a network dimensioning approach that can be adapted to the specific qualities of various PON technologies. The split ratio (number of homes passed by fiber from a single OLT PON interface) is optimized to use the PON capacity of each technology as effectively as possible, taking into account user demands. Moreover, we model the optimal deployment of COs in a major city, taking into account technology-dependent reach constraints, as the geographic spread of the locations may impact the filling ratio of OLT racks. Our user demand 
model considers triple-play services, consisting of (1) fixed voice, (2) high definition Internet protocol television (IPTV), and (3) best-effort Internet and over the top (OTT) media access with up to $1 \mathrm{~Gb} / \mathrm{s}$ download speed per customer. The model presented in this paper builds on our earlier work [7, 8]; the main differences are the inclusion of IPTV services, general OLT functions now include packet processing and traffic management, updated estimates for OLT and ONU power consumption and an in-depth sensitivity analysis.

Concretely, the energy efficiency analysis is implemented as follows (Fig. 1). We start by choosing a number of interesting cases to study. Each case consists of a specific user demand (access speed, number of subscribers,...), deployment strategy (one of two options: fixed or optimized split ratio) and PON technology (one of seven options introduced in Section 3). Next, a three-stage power consumption analysis is performed for each case. In the first stage, the PON is dimensioned, based on the technology-dependent reach and capacity, user demands and QoS requirements. The PON dimensioning approach is described in Section 4. It produces the requirements for uplink and general OLT functions (switching, packet processing, traffic management), which are used further on for the power consumption calculation. It also produces the split ratio, which is used as an input for the second stage: the city deployment algorithm, which is covered in Section 5. The algorithm contains a model of the geographical distribution of homes in a major European city, and calculates how much equipment needs to be installed, given the takerate (i.e., the number of fiber subscriptions divided by the number of homes passed by fiber), the technology-dependent reach and the split ratio (depending on the case under study). This stage returns the equipment count (number of ports, racks,...), which is an input for the third and last stage: the power consumption calculation. The power consumption calculation is introduced first in this paper, in Section 2 , because it provides a good overview of the access network and its components, and it drives the calculations in the previous stages.

Section 6 presents the results for a number of selected cases and compares the energy efficiency of the various PON technologies. Section 7 contains a sensitivity analysis, to determine which parameters are most critical to the energy efficiency model. Finally, a summary of the results and the conclusions of the work are reported in Section 8.

\section{Power consumption model for the access network}

Figure 2 gives a schematic overview of the considered optical access network. It comprises the optical network unit (ONU) at the customer premises, the optical line terminal (OLT) and uplink to the aggregation network at the network operator's $\mathrm{CO}$ and the fibers and splitters between them (optical distribution network or ODN). Since we evaluate passive optical networks in this paper, no active equipment is needed in the ODN. Moreover, only technologies over an ODN with optical power splitters will be considered (no wavelength-selective components), thus ensuring full coexistence between several PON generations using the same passive technology, without the need for modifying or replacing the already existing components in the outside plant.

Our power consumption model is mostly based on models and values that are (were) available within the projects GreenTouch and Trend, combined with values reported by Skubic et al. in [2].

\subsection{Power per user}

We consider a deployment in a city where $H$ homes are subscribing to the PON. The total power consumption of the citywide access network is the sum of the consumption of all ONUs and all OLTs, $P_{\text {ONUs }}$ and $P_{\text {OLT }}$ respectively. To obtain the power per user, we simply divide the total power by the number of subscribers.

$$
\text { Total power }[\text { W/user }]=\frac{P_{\text {ONUs }}+P_{\text {OLTs }}}{H}
$$

\subsection{City-wide power consumption by $O N U s$}

Because we consider a fiber to the home (FTTH) scenario, each subscribing home has its dedicated ONU. All ONUs have a common baseline power dissipation $P_{\text {baseline }}$ of $3.65 \mathrm{~W}$. This baseline power consists of contributions from the embedded processor, gigabit Ethernet interface, dual subscriber line interface circuit (SLIC), memory, and other miscellaneous components [2]. The technology-dependent ONU transceiver power $P_{\text {ONU,tech }}$ (see Table 1) is added to this baseline, and the total is multiplied by a factor 1.25 to account for AC/DC (Alternating Current/Direct Current) rectifier and DC/DC (Direct Current/Direct Current) voltage conversion efficiencies $\left(\eta_{A C / D C}=\right.$ $\left.\eta_{D C / D C}=90 \%\right)$

$$
P_{\text {ONUs }}=H \times\left(P_{\text {baseline }}+P_{O N U, \text { tech }}\right) \times \frac{1}{\eta_{A C / D C}} \times \frac{1}{\eta_{D C / D C}}
$$

\subsection{City-wide power consumption by OLTs}

On the OLT side, the power consumption is the sum of the contributions from OLT PON ports, general OLT functions (switching, packet processing and traffic management), and the uplinks to the aggregation network. On top of a factor 1.11 for DC/DC conversion inefficiency, we multiply by a site factor 1.6 at the $\mathrm{CO}$ to account for auxiliary equipment such as AC/DC rectifiers $\left(\eta_{A C / D C}=90 \%\right)$, ventilation and air conditioning, auxiliary power units and batteries [2].

$P_{\text {OLTs }}=\left[P_{\text {ports }}+P_{\text {gen. func. }}+P_{\text {uplinks }}\right] \times \frac{1}{\eta_{D C / D C}} \times \mathrm{CO}$ site factor

The power consumption of the OLT PON ports is technology-dependent and consists of a power per PON port $P_{\text {port,tech }}$ and, for some technologies, an added power per user $P_{\text {user,tech }}$ (see Table 1). The power per port is multiplied by the number of ports in the city-wide deployment (obtained in Section 5), and the power per user is multiplied by the number of subscribers.

$$
P_{\text {ports }}=\# \text { ports } \times P_{\text {port }, \text { tech }}+H \times P_{\text {user,tech }}
$$




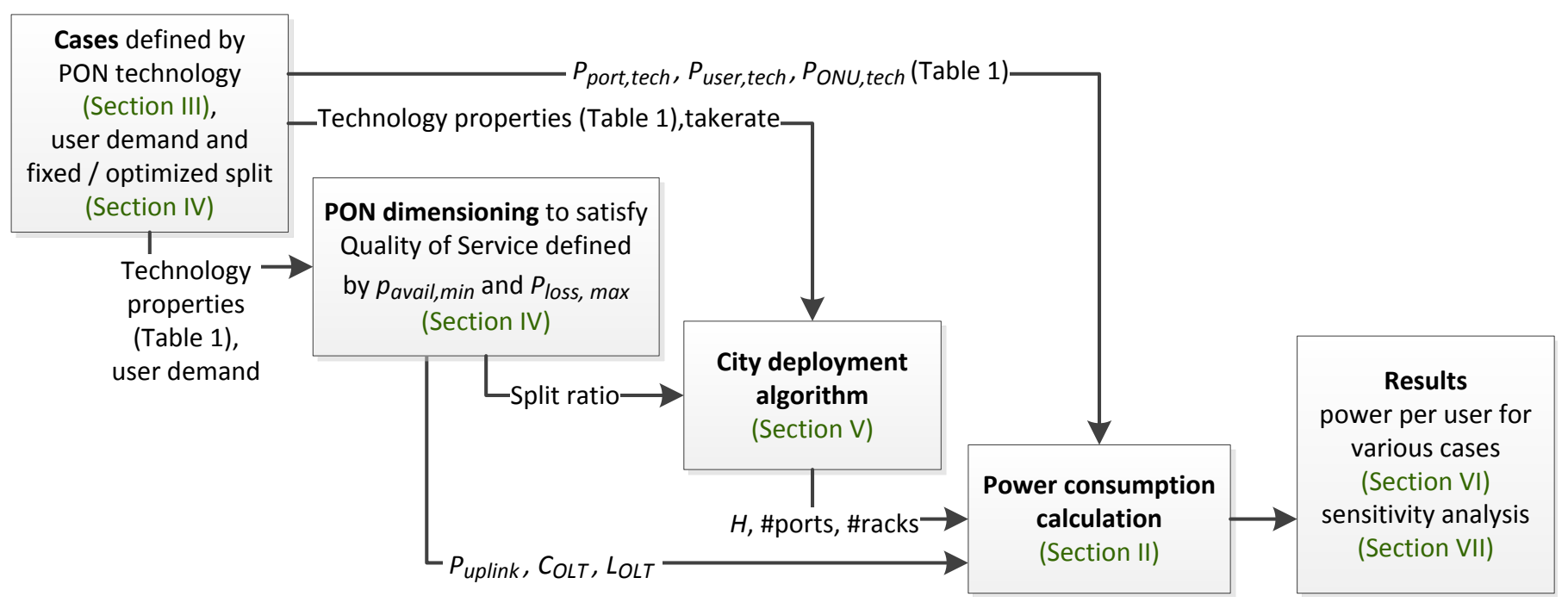

Figure 1: Overview of methodology and paper contents (symbols introduced in Section 2).

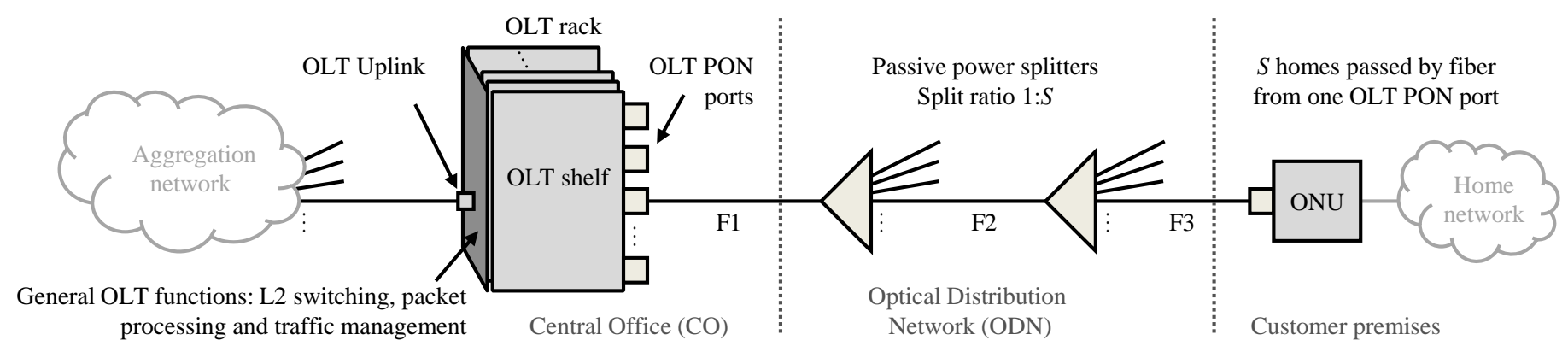

Figure 2: Schematic overview of the access network and its power consumption components. F1, F2, F3 = optical fiber sections. 
The city-wide power consumption of the general OLT functions and uplinks is obtained by multiplying the total number of racks in the city (obtained in the city deployment model, Section 5) by the power per rack, which depends on the capacity and traffic load per rack. The average traffic load $L_{O L T}$ and the required capacity $C_{O L T}(\mathrm{~Gb} / \mathrm{s})$ are dimensioned for a single rack in Section 4.3.

For the general OLT functions, a unidirectional power of $1 \mathrm{~W} / \mathrm{Gb} / \mathrm{s}$ is assumed, consisting of $0.5 \mathrm{~W} / \mathrm{Gb} / \mathrm{s}$ for Layer 2 switching [9] and $0.5 \mathrm{~W} / \mathrm{Gb} / \mathrm{s}$ for packet processing and traffic management. $30 \%$ of this power scales with the required capacity, the other $70 \%$ scales with the actual traffic load (for details, see Section 4.3.2).

$$
P_{\text {gen. func. }}=\# \text { racks } \times\left(0.3 \times C_{O L T}+0.7 \times L_{O L T}\right) \times 1 \mathrm{~W} / \mathrm{Gb} / \mathrm{s}
$$

The uplink of a rack is formed by a combination of Ethernet ports (with corresponding power consumption values given in Section 4.3.1), dimensioned such that for a given user traffic, the packet loss in the uplink remains acceptable.

$$
P_{\text {uplinks }}=\# \text { racks } \times P_{\text {uplink }}=\# \text { racks } \times \sum P_{\text {Ethernet port }, i}
$$

In the following section, we will take a closer look at the PON technologies and their specific parameters. Further on we will discuss the PON dimensioning approach that produces the split ratio and OLT requirements, and the city deployment model that produces the equipment inventory.

\section{Overview of the considered PON technologies}

In this section, we start by giving a brief description of the seven technologies included in our power consumption comparison. Next, we expand on the technology-specific physical limitations (optical budget and bandwidth) and power consumption values.

\subsection{Technologies considered in this paper}

The commercially available Gigabit PON (GPON) system with $\mathbf{B}+$ optics is used as reference technology in this work. Next-generation PON technologies are considered, including $10 \mathrm{~Gb} / \mathrm{s}$ PON (XG-PON1 E2 class) as well as several candidates of the second generation systems (NG-PON2) with at least $40 \mathrm{~Gb} / \mathrm{s}$ capacity per PON. The technological framework of NG-PON2 systems has been described by the FSAN (Full Services Access Network) group [1]. In this paper, we focus on the following NG-PON2 technologies:

- 40Gbit/s capable (XLG) PON, which consists of a time division multiplexed (TDM) PON using a single wavelength in downstream (DS) with $40 \mathrm{~Gb} / \mathrm{s}$ line rate and a single wavelength in upstream (US) for all ONUs with $10 \mathrm{~Gb} / \mathrm{s}$ line rate. O-band DS transmission is assumed to avoid dispersion compensation. We consider two varieties of this technology, with different protocols for DS transmission. XLG:GEM uses the GPON Encapsulation Method
(GEM) to package user traffic. The payload is grouped into frames, which are filtered at the ONU based upon the GEM header. This requires electronic processing of the incoming traffic bursts in the ONU at $40 \mathrm{~Gb} / \mathrm{s}$. XLG:BI uses an alternative bit-interleaving protocol to transmit DS traffic. By interleaving the bits for different ONUs, the electronic processing speed of the ONU receiver can be reduced, resulting in a lower ONU energy consumption [10].

- Time-shared Wavelength Division Multiplexing (TWDM) PON consists of four overlaid TDM-PONs in a single physical ODN, using different wavelengths both in DS and US directions, each TDM-PON with a $10 \mathrm{~Gb} / \mathrm{s}$ line rate in DS and $2.5 \mathrm{~Gb} / \mathrm{s}$ in US. Tunable ONU transmitters and receivers are assumed, to distribute the subscribers evenly among the four wavelengths (in this work, tunability is not used for dynamic bandwidth allocation in response to variations in user activity).

- Orthogonal Frequency Division Multiplexing (OFDM) PON, where multiple orthogonal electrical carriers are multiplexed. The ONU can flexibly filter and downconvert a band of $N_{s}$ subcarriers in the analogue domain ${ }^{1}$, so that subsequent digital signal processing (DSP) and media access control (MAC) functions can be performed at a lower rate.

- Coherent Ultra Dense WDM-PON (Co UDWDM), consisting of a logical point-to-point dense WDM-PON system with tunable ONUs and coherent detection [11], which achieves the highest optical power budget. Though systems supporting over a thousand wavelengths in the same PON have been reported, a maximum of 256 wavelengths is considered in this paper. In any case, the impact on the power/user of adding more wavelengths would be limited, since the main contribution to the OLT power for Co UDWDM comes from the added power per user (cf. Section 3.3).

FSAN selected TWDM-PON as the main technology for NGPON2, with an expected practical availability by 2015-2016. The other next-generation technologies that we consider in this paper should be considered for future evolution beyond NGPON2.

\subsection{System parameters}

Table 1 shows the aggregated bandwidth capacity per PON interface in DS and US directions, the number of PON ports per OLT rack, the maximum optical power budget, and attenuation for each PON technology. In order to calculate the maximum reach for each technology, $0.6 \mathrm{~dB} / \mathrm{km}$ propagation losses are assumed for the $\mathrm{O}$ band and $0.4 \mathrm{~dB} / \mathrm{km}$ for the $\mathrm{C}$ and $\mathrm{L}$ bands, comprising the typical average losses of splices and other penalties in the fiber outside plant.

\footnotetext{
${ }^{1}$ One orthogonal subcarrier for each of the $N_{s}$ subscribers in the PON.
} 
Table 1: Technology-dependent system and power consumption parameters.

\begin{tabular}{|c|c|c|c|c|c|c|c|}
\hline PON technology & $\begin{array}{c}\text { DS capacity } \\
(\mathrm{Gb} / \mathrm{s})^{\mathrm{a}}\end{array}$ & $\begin{array}{c}\text { US capacity } \\
(\mathrm{Gb} / \mathrm{s})^{\mathrm{a}}\end{array}$ & $\begin{array}{l}\text { PON ports } \\
\text { per rack }\end{array}$ & $\begin{array}{l}\text { Max. } \\
\text { optical } \\
\text { budget } \\
(\mathrm{dB})\end{array}$ & $\begin{array}{l}\text { Attenua- } \\
\text { tion } \alpha \\
(\mathrm{dB} / \mathrm{km})\end{array}$ & $\begin{array}{c}\text { Power per OLT } \\
\text { PON port }(\mathrm{W})^{\mathrm{a}, \mathrm{b}} \\
P_{\text {port tech }}+ \\
P_{\text {user,tech }} \times N_{s}\end{array}$ & $\begin{array}{l}\text { Power per } \\
\text { ONU }(W)^{b}\end{array}$ \\
\hline GPON B+ & 2.5 & 1.25 & 256 & 28.0 & 0.6 & 3.0 & 2.6 \\
\hline XG-PON1 & 10 & 2.5 & 128 & 35.0 & 0.6 & 22.8 & 4.5 \\
\hline XLG:GEM & 40 & 10 & 64 & 31.0 & 0.6 & 36.1 & 6.1 \\
\hline XLG:BI & 40 & 10 & 64 & 31.0 & 0.6 & 36.1 & 4.6 \\
\hline TWDM & $4 \times 10$ & $4 \times 2.5$ & 64 & 35.0 & 0.4 & 54.8 & 5.0 \\
\hline OFDM & 40 & 10 & 64 & 34.5 & 0.6 & $42.8+0.7 \times N_{s}$ & 9.3 \\
\hline Co UDWDM & $1.25 \times N_{s}$ & $1.25 \times N_{s}$ & 64 & 43.0 & 0.4 & $9.3+3.5 \times N_{s}$ & 5.7 \\
\hline
\end{tabular}

${ }^{\text {a }} N_{s}=$ number of subscribers per OLT PON port. $N_{s}$ scales with the split ratio and takerate.

$\mathrm{b}$ Power consumption values are system-specific contributions, excluding common baseline for ONUs and not yet taking into account conversion inefficiencies and site factor.

\subsection{Power consumption parameters}

Estimates of the power consumption for system-specific electro-optical components per OLT PON port (consisting of a per-PON and an optional per-user component) and per ONU, are reported for each technology in the last two columns of Table 1 . The power values reflect how much a practical ASIC implementation of the PON system would consume if it were made today. Future improvements in electronic and optical design will likely result in reduced power consumption, but these overall improvements will not impact the comparison between technologies. For NG-PON2 technologies, which are not yet commercially available, the power consumption values are best-effort estimates based on internal data and values from literature $[12,13,2]$. The impact of uncertainty in these estimates is discussed in the sensitivity analysis (Section 7).

The power consumption per OLT PON port increases as the PON bandwidth increases. For OFDM and Co UDWDM PON technologies, a variable part of the OLT port power consumption scales with the number of users, considering the additional power contribution of digital processing and transceiver groups required when more users are connected to the PON. ${ }^{2}$ Except for GPON systems, amplified solutions have been considered for long reach capabilities. An SOA (semiconductor optical amplifier) for both DS and US direction is included in the OLT numbers for XG, TWDM, OFDM and Co UDWDM PON. For XLG-PON, we consider DS amplification by means of an SOA, and electronic dispersion compensation for the US signal (XLG:GEM and XLG:BI use identical equipment on the OLT side).

Significant differences can be observed for the power consumption of the ONU. The technology-specific component typically increases proportionately with the line rate. However, the ONU of a XLG:BI PON can consume about the same power

\footnotetext{
${ }^{2}$ For Co UDWDM, we assume a pay-as-you-grow concept, where the network operator installs additional capacity as needed.
}

as XG-PON1, despite a four times higher line rate, by using an energy efficient bit-interleaving protocol in the DS direction [10]. The TWDM PON ONU consumes slightly more power than XG-PON1 because it is based on the same standard MAC protocol and offers the same line rate, but consumes additional power for the tuning of the laser and receive filter. OFDM PON is highly inefficient due to the need for DSP and optical amplification to meet the stringent signal to noise ratio across a standard ODN. Even though we assumed the possibility to select a subset of carriers and as such reduce the power consumption of the DSP and MAC processing, the ONU power consumption remains high. The optical front-end of the ONU in a coherent UDWDM PON consumes more power due to the coherent receiver requiring two balanced receiver pairs and the optical field modulator for US transmission. On the other hand, the protocol processing is simplified to a $1 \mathrm{~Gb} / \mathrm{s}$ Ethernet functionality, which consumes less power than in an XLG PON or TWDM PON. The total power of a Co UDWDM ONU is thus only slightly higher than that of an XLG PON or TWDM PON ONU.

\section{PON dimensioning based on user demand}

In this section, we explain how the PON is dimensioned based on a statistical analysis of the aggregated user demands. This statistical analysis takes into account the potential for dynamic bandwidth allocation. The extent to which bandwidth can be shared among users, depends on the chosen PON technology. Therefore, we introduce the concept of a virtual PON, in which bandwidth can be divided arbitrarily between the active users. For GPON, XG-PON, XLG:GEM, XLG:BI and OFDM PON, the virtual PON corresponds to the physical PON. In the case of TWDM PON, each physical PON consists of four virtual PONs on separate wavelengths. In a Co UDWDM PON, users have their own dedicated wavelength channel, resulting in as many virtual PONs as there are users, so there is no dynamic 
bandwidth allocation between users in this case. We consider line rates for the definition of the access speed per subscriber, assuming a similar level of overhead for all PON technologies.

When dimensioning the PON, there are three types of user demands we considered: voice traffic, multicast IPTV traffic and best-effort Internet traffic (including OTT Internet video). The dimensioning for IPTV multicast demands and the statistical flow analysis for best-effort Internet traffic will be performed per virtual PON. In our traffic analysis, we only model DS bandwidth, assuming US traffic is less than or equal to $25 \%$ of DS traffic.

\subsection{User demand model for triple play services}

Voice traffic is the highest priority traffic in case of congestion, so the consumed bandwidth by voice should be subtracted from the total bandwidth available for best-effort Internet and IPTV services. The speed rate of VoIP codecs ranges from $22 \mathrm{~Kb} / \mathrm{s}$ to $113 \mathrm{~Kb} / \mathrm{s}$ [14], which is less than $0.1 \%$ of the total access bandwidth values considered in this work, and the average minutes of use per user of fixed voice is 10 minutes per day [15], thus the contribution of voice to the traffic load is negligible and has not been included in the simulations. Nevertheless, in real implementations, this VoIP traffic will need to have the highest priority.

Multicast IPTV traffic also has priority over best-effort Internet traffic. Note that we focus on IPTV in the context of traditional broadcast live TV, as opposed to Video on Demand, which is considered part of the (OTT) best-effort Internet traffic. To estimate the number of channels being watched, we base ourselves on IPTV channel popularities from measured weekly viewing times for a commercial IPTV service in the United Kingdom. We assume HD channels with MPEG4 encoding (12 Mb/s per channel). All IPTV channels (127 in total) are broadcast from the aggregation network to the OLTs through the uplinks, but only the channels that are being watched by users within a (virtual) PON are forwarded via multicast within that PON. To guarantee IPTV transmission, we estimate the bandwidth that is required for multicast within a virtual PON during primetime, and reserve it, making it unavailable for besteffort Internet services. We assume $25 \%$ of Internet subscribers have an IPTV subscription, and each TV subscriber has 1.85 TVs on average ${ }^{3}$, of which $60 \%$ are switched on during primetime. We use a Monte Carlo approach to assess the number of TV channels that need to be distributed per virtual PON, similar to the approach used by van Veen et al. in [17]; we however use the measured channel popularities, whereas van Veen et al. modeled channel popularity with a Zipf function. We reserve enough bandwidth in each PON to ensure IPTV multicast is uninterrupted $99.9 \%$ of the time ${ }^{4}$. In our results, IPTV multicast bandwidth per PON is always below $15 \%$ of the total PON bandwidth.

${ }^{3} 40 \%$ of TV subscribers have one TV set, $35 \%$ have two, and $25 \%$ have three [16].

${ }^{4}$ We assume the operator does not allow fast channel surfing (mean time between channel changes $<4$ seconds) as this would result in a much higher load during commercial breaks, up to twice the steady state level [18].
The bulk of the traffic is best-effort Internet traffic. In our Internet service model, a maximum target bandwidth, namely $B_{\text {target }}$, is offered to each customer with a minimum percentage of time of availability, namely $p_{\text {avail,min }}$. We adopt the user behavior model from Segarra et al. [19], where each user has the same probability $p_{a c t}$ to be active and users are independent. ${ }^{5}$ In our simulations, $p_{\text {act }}$ is fixed at $10 \%$ [20]. We extend the model from Segarra et al. by assuming that users request a fixed target bandwidth $B_{\text {target }}$ when they are active. Fastest average connections offered by operators already reached $1 \mathrm{~Gb} / \mathrm{s}$ in 2012 [21], thus we consider three possible regular access speeds for the majority of users in future broadband scenarios: $B_{\text {target }}=100 \mathrm{Mb} / \mathrm{s}, 600 \mathrm{Mb} / \mathrm{s}$ and $1 \mathrm{~Gb} / \mathrm{s}$.

The service models for IPTV and best-effort Internet described above, will be used in the next subsections for the split ratio and OLT dimensioning.

\subsection{Split ratio dimensioning}

Two different deployment strategies are used in this work to compare the seven PON technologies. The first is a fixed split ratio strategy, in which an existing GPON deployment with split ratio 1:64 is re-used by upgrading the ONUs and OLTs, without any changes in the ODN. For each technology, we calculate $p_{\text {avail }}$ using the method described in subsection 4.2.2 below, to check if the QoS requirement is satisfied. The second strategy assumes a fully flexible deployment, in which an optimized split ratio is chosen. We determine the maximal split ratio at which $p_{\text {avail,min }}$ can be guaranteed. Note that this optimized split ratio may be higher or lower than the legacy value of 1:64, depending on takerate, user demand, offered bandwidth and PON technology. There is a trade-off between availability (QoS) and power consumption: increasing the split ratio reduces availability, but it also reduces power consumption as the OLT equipment is shared by more users. We assume a maximum split ratio 1:256, with additional restrictions imposed by the technology-dependent reach.

\subsubsection{Reach restrictions}

The reach of a technology for a given physical split ratio $1: S$ is

$$
d_{\max }=\frac{\left(\text { max. optical budget }-L_{m}-L_{c}-3.5 \times \log _{2} S\right)}{\alpha}
$$

where $L_{m}$ is a $3 \mathrm{~dB}$ margin for fiber patching (based on practical experience) and $L_{c}$ is a $1 \mathrm{~dB}$ penalty for a coexistence element in the COs, used for compatibility with existing GPON deployments (based on ITU-T Rec. G.984.5 Amd. 1). Note that $L_{c}$ is zero when calculating the reach for GPON. Splitter losses scale with the chosen split ratio: the signal incurs a $3.5 \mathrm{~dB}$ loss for each doubling of the split ratio (assumption adopted from OASE [9], consistent with ITU-T Rec. G.671). The maximum

${ }^{5}$ Users are not perfectly independent in real networks, as some periods of the day will be more busy than others. The assumptions made here are to be interpreted as estimates for peak hours. 
optical budget and attenuation factor $\alpha$ for each technology are listed in Table 1. In our calculations, we limit ourselves to those subsets of split ratios that allow a reach $d_{\max }$ that is greater than $5 \mathrm{~km}$, to ensure all homes in the city can be connected to the COs.

\subsubsection{Quality of Service $(Q o S)$ restrictions}

After subtracting the IPTV multicast bandwidth (calculated in Section 4.1) from the total PON bandwidth, the remaining best-effort Internet bandwidth is to be shared by all subscribers in the (virtual) PON. We assume ideal dynamic bandwidth allocation without packet loss in the ODN in both US and DS direction. All users within a virtual PON are treated equally. When $k$ active users from $N$ total independent users are demanding or delivering traffic from/to an OLT interface, the maximum bandwidth that can be offered to each user is

$$
B_{\text {max }}=\frac{\text { PON bandwidth }}{k}, 1 \leq k \leq N
$$

When $B_{\max }$ is greater than $B_{\text {target }}$, all active users get the requested $B_{\text {target }}$. However, when there are too many active users, $B_{\text {max }}$ may be smaller than $B_{\text {target }}$, so the offered bandwidth may be lower than the requested bandwidth. The probability $p_{\text {avail }}$ that $B_{\text {target }}$ is available ( $B_{\max } \geq B_{\text {target }}$ ), equals the probability that the number of active users $k$ is smaller than or equal to $k_{\text {max }}$, given by the cumulative binomial probability [19]

$$
\begin{aligned}
p_{\text {avail }} & =\sum_{k=0}^{k_{\max }} \frac{N !}{(N-k) ! k !}\left(1-p_{\text {act }}\right)^{N-k} p_{\text {act }}^{k} \\
k_{\text {max }} & =\left\lfloor\frac{\text { PON bandwidth }}{B_{\text {target }}}\right\rfloor
\end{aligned}
$$

This availability is compared to $p_{\text {avail,min }}$, the minimum percentage of time that the target bandwidth should be available for each connected user ( $p_{\text {avail,min }}=90 \%$ in our simulations, based on the current average for residential fiber services [22]). In case of the fixed split ratio approach, this may eliminate technologies that cannot meet QoS requirements. In case of split ratio optimization, the split ratio is lowered until QoS requirements are met.

\subsection{OLT dimensioning for a single rack}

Once the split ratio has been determined, we can dimension the general OLT functions and the uplink between the OLT and the aggregation network. The traffic passing through an $\mathrm{OLT}^{6}$ consists of three contributions: (1) Voice traffic: requires negligible bandwidth, (2) IPTV broadcast of all TV channels, for which a combined downstream capacity of $127 \times 12 \mathrm{Mb} / \mathrm{s}=1.5 \mathrm{~Gb} / \mathrm{s}$ is reserved, and (3) best-effort Internet traffic: the dimensioning for this traffic is derived from the user demands as follows.

In our model, the number of active users in a single PON follows a binomial distribution (see Section 4.2.2). By multiplying the numbers of active users in this distribution by

\footnotetext{
${ }^{6}$ Here, "OLT" refers to a single rack, connecting a number of PONs as listed in Table 1.
}

$B_{\text {target }}$, and capping the values at the available PON capacity for best-effort Internet services, we obtain the demand distribution $\left(D_{P O N}, P_{P O N}\right)$ for a single (virtual) PON. This distribution is used in a Monte Carlo simulation, where for each PON in the rack, a random number is generated and mapped onto a demand $D_{P O N}(j)$ with probability $P_{P O N}(j)$, and the demands of the PONs in the rack are added up. After running the simulation one million times, we can estimate the combined best-effort Internet traffic load of the PONs connected to a rack $\left(D_{O L T}, P_{O L T}\right)$ with sufficient precision. Based on this distribution, we dimension the uplink and general OLT functions requirements per rack for best-effort Internet services as described in the following sections.

\subsubsection{Uplink}

The uplink capacity should be sufficiently high so as to keep the packet loss in the uplink $\left(P_{\text {loss }}\right)$ unnoticeable for users. Average packet loss in present-day residential fiber access networks is in the range $0.13 \%-0.34 \%$ [22], so we decide to keep the packet loss in the uplink below $P_{\text {loss, } \max }=0.1 \%$ for the reference scenario. Transmission Control Protocol (TCP) connections with high sustainable rates up to $1 \mathrm{~Gb} / \mathrm{s}$ may require a lower packet loss rate, in which case a more strict value can be chosen without impacting the conclusions of this work, as the sensitivity analysis shows that overall power consumption is not impacted significantly by varying $P_{\text {loss, } \max }$ from $10^{-3}$ to $10^{-8}$.

We calculate the minimal uplink capacity $C_{U}$ for which $P_{\text {loss }}<P_{\text {loss,max }}$ as follows. In the uplink interface of an OLT rack, the packet loss is the ratio of packets discarded over packets offered. We use the flow model from [23] to estimate packet loss: instead of considering individual packets, we look at traffic flows. The packet loss is then

$$
P_{\text {loss }}=1-\frac{m}{m^{*}}
$$

where $m^{*}$ is the average combined traffic load from all PONs connected to the rack (traffic offered), and $m$ is the mean traffic load passing through the uplink (traffic passed). For a given uplink capacity $C_{U}$ for best-effort Internet services, $m^{*}$ and $m$ are easily derived from the OLT load distribution as follows

$$
\begin{aligned}
m^{*} & =\sum_{i} D_{O L T}(i) \times P_{O L T}(i) \\
m & =\sum_{i} \min \left(D_{O L T}(i), C_{U}\right) \times P_{O L T}(i)
\end{aligned}
$$

The minimal uplink capacity $C_{U}$ for which the packet loss calculated in (11) is below $P_{\text {loss,max }}$, is chosen. This capacity $C_{U}$ is then added to the IPTV broadcast bandwidth $(1.5 \mathrm{~Gb} / \mathrm{s})$ to obtain the total required uplink capacity.

The uplink is realized using a combination of Ethernet ports with capacities and corresponding power consumption values adopted from [24]: $1 \mathrm{~Gb} / \mathrm{s}, 10 \mathrm{~Gb} / \mathrm{s}, 40 \mathrm{~Gb} / \mathrm{s}, 100 \mathrm{~Gb} / \mathrm{s}$, $400 \mathrm{~Gb} / \mathrm{s}$ and $1 \mathrm{~Tb} / \mathrm{s}$ ports consume $7 \mathrm{~W}, 38 \mathrm{~W}, 105 \mathrm{~W}, 205 \mathrm{~W}$, $560 \mathrm{~W}$ and $1100 \mathrm{~W}$ respectively. The port combination with minimal energy consumption is chosen to realize the uplink. 
Note that only downstream traffic is considered in this calculation, since the uplink ports are symmetrical and there is typically more traffic load downstream than upstream.

\subsubsection{General OLT functions}

The last contribution to the OLT power consumption comes from the general OLT functions, which include (1) Layer 2 (Ethernet) switching, and (2) packet processing and traffic management. About $30 \%$ of this power consumption is static, due to leakage in transistors, and scales with capacity; the other $70 \%$ is dynamic and scales with the actual traffic load.

Half of the static power consumption is used for switching, for which the installed capacity is symmetrical and thus scales with the highest traffic capacity direction (downstream); the other half is used for packet processing and traffic management, for which different upstream and downstream capacity can be installed. So the OLT capacity $C_{O L T}$ in equation (5) should be interpreted as $0.5 \times\left(2 \times C_{D S}\right)+0.5 \times\left(C_{D S}+C_{U S}\right)$ to differentiate between these symmetrical and asymmetrical contributions. The required downstream capacity $C_{D S}$ of the general OLT functions is the sum of IPTV broadcast capacity $(1.5 \mathrm{~Gb} / \mathrm{s})$ and DS best-effort Internet capacity of the OLT. The upstream OLT capacity $C_{U S}$ is $25 \%$ of the DS best-effort Internet capacity. The DS best-effort Internet capacity is estimated using a similar method as for the uplink capacity, but with a stricter limitation on the allowable packet loss $\left(10^{-9}\right)$. Moreover, this capacity is multiplied by a factor four to overdimension the equipment to accommodate traffic growth and traffic peaks (an average factor which we obtained as a rule of thumb from providers). The assumptions for OLT capacity dimensioning are more strict than those for the uplink because it is more difficult to upgrade OLT capacity: for the uplink, capacity can be increased by simply plugging in additional transceivers and adding capacity in the aggregation switches in the metro aggregation net, whereas an OLT upgrade will likely involve a replacement of the OLT switch and packet processors.

The dynamic power consumption is proportional with the traffic load, and consumes $0.70 \mathrm{~W} / \mathrm{Gb} / \mathrm{s}$ (unidirectional, consisting of $0.35 \mathrm{~W} / \mathrm{Gb} / \mathrm{s}$ for switching and $0.35 \mathrm{~W} / \mathrm{Gb}$ for packet processing and traffic management). This value is multiplied by the average OLT traffic load $L_{O L T}$, which is the sum of the average downstream best-effort Internet traffic load, $25 \%$ of this value for upstream traffic, and the IPTV broadcast bandwidth.

\section{City deployment model}

A real city scenario is considered in the PON deployment algorithm which - based on the technology-dependent optical budget, attenuation, split ratio and number of PON ports per rack - finds the required inventory of active equipment for providing high speed triple play services with the required QoS. ${ }^{7}$ A greenfield approach is followed for fiber network construction, assuming that no other optical access infrastructure has

\footnotetext{
${ }^{7}$ City deployment algorithm developed in the framework of FP7 project Accordance.
}

Table 2: Summary of city topology.

\begin{tabular}{|c|c|c|c|c|}
\hline & $\begin{array}{c}\text { Central } \\
\text { zone }\end{array}$ & Ring 1 & Ring 2 & Ring 3 \\
\hline $\begin{array}{l}\text { Population } \\
\text { centers }\end{array}$ & 1 & 13 & 19 & 40 \\
\hline Home units & 564,730 & 434,376 & 276,217 & 165,903 \\
\hline $\begin{array}{l}\text { Density } \\
\text { threshold } \\
\text { (homes } / \mathrm{km}^{2} \text { ) }\end{array}$ & 4,000 & 4,000 & 2,000 & 500 \\
\hline $\begin{array}{l}\text { Fiber length } \\
\text { F1, F2, F3 }\end{array}$ & $\begin{array}{l}40 \% \\
20 \% \\
50 \mathrm{~m}\end{array}$ & $\begin{array}{l}40 \% \\
20 \% \\
50 \mathrm{~m}\end{array}$ & $\begin{array}{l}42 \% \\
23 \% \\
75 \mathrm{~m}\end{array}$ & $\begin{array}{l}44 \% \\
26 \% \\
100 \mathrm{~m}\end{array}$ \\
\hline Surface $\left(\mathrm{km}^{2}\right)$ & 67.27 & 91.80 & 113.03 & 178.81 \\
\hline $\begin{array}{l}\text { Distance to } \\
\text { central zone } \\
(\mathrm{km})\end{array}$ & 0 & 15.55 & 23.11 & 29.21 \\
\hline
\end{tabular}

${ }^{\text {a }}$ Values for F1, F2 and F3 are based on a typical real GPON deployment. F1 and F2 are reported as percentages of min(population center radius, physical reach of the PON technology) to account for reach differences between PON technologies.

been deployed before and if there are existing ducts, they are not necessarily used. This allows to evaluate the impact of the different PON deployment parameters with total flexibility. In case of a fixed split ratio approach, we assume the existing deployment has been optimized in the past using the same algorithm to obtain the equipment count.

\subsection{City description}

Public source real data from the Spanish National Institute of Statistics [25] have been employed to create the city topology model. This work focused on a major city area in Spain with an aggregate distribution of 1.5 million home units. A classification of the 73 population centers of the city was established using three geotypes depending on population density: (1) Dense urban geotype with $\geq 4,000$ home units per $\mathrm{km}^{2}$, (2) Urban geotype with 2,000 - 4,000 home units per $\mathrm{km}^{2}$, and (3) Sub-urban geotype with $500-2,000$ home units per $\mathrm{km}^{2}$. A simplified model of the city area was built considering three concentric rings with a common central zone. Both the central zone and Ring 1 correspond to the dense urban geotype, while Ring 2 and 3 correspond to urban and sub-urban geotypes, respectively (see Table 2).

\subsection{Optical Access Network Deployment}

For a given service definition, the number of OLT racks depends on the filling ratio of each OLT PON interface, which is based on two factors: (1) the coverage area of a PON, which depends on its maximum physical reach, determined by the split ratio, optical budget and attenuation; and (2) the population density, determined by its location in the city. As a con- 

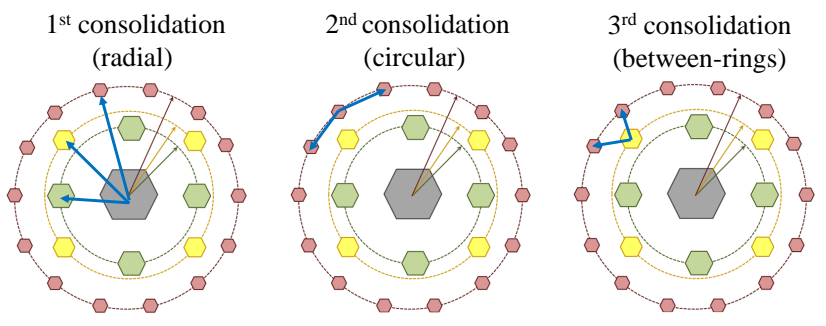

Figure 3: Graphical representation of the CO consolidation steps.

sequence, the first step for the PON inventory calculation is locating the COs. Initially, one $\mathrm{CO}$ is associated to each of the 73 population centers. For simplicity, each of the rings' surfaces is evenly assigned to each of the identified population centers, and all of the COs of each ring are considered to be at the average distance with regards to the central area. Next, a concentric $\mathrm{CO}$ consolidation algorithm is used as PON deployment optimization criterium, because it allows minimum real estate investment for the network operator, as well as lower operational cost. Considering two levels of power splitting in the PON fiber outside plant, the rules for fiber network construction to provide FTTH coverage depending on each type of population center are shown in Table 2 (fiber sections F1, F2 and F3 as indicated in Fig. 2). For a given PON technology, the algorithm used to calculate the number of COs and the ring location of each $\mathrm{CO}$ consists of the following three steps (see Fig. 3):

1. Radial consolidation. For each of the three rings, the algorithm verifies if an extended feeder fiber from the central zone to the average ring distance is supported, depending on the reach of the PON technology. If the verification is positive, then all the home units of the verified ring are served from the central zone.

2. Circular consolidation. When radial consolidation is not possible for a ring, then consolidation of neighboring COs into a single $\mathrm{CO}$ within the same ring zone is verified. An average distance between COs is calculated from the ring area and the number of centers in the ring. Increasing multiples of this average distance are added to the length of fiber section F1 for the corresponding ring and the maximum circular consolidation is obtained considering the reach of the PON technology.

3. Inter-ring consolidation. After the circular consolidation, the algorithm verifies if the remaining COs in a ring, which cannot be served from the previously consolidated COs (isolated centers), can serve centers of an outer ring.

Thus, depending on the technology performance and the service requirements, the number of serving COs in each of the rings is calculated and the corresponding equipment inventory for the COs (number of OLT racks and OLT PON ports) is returned. The rack filling rate - the actual number of PON ports per rack divided by the theoretical number of PON ports per rack - will depend on the location of the COs, and has an impact on the total power consumption of the access network.

The number of ONUs is also calculated in this stage, based on the number of homes in the city, the percentage of real estate

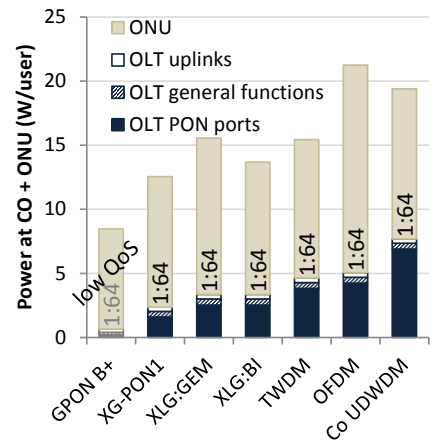

(a) fixed split

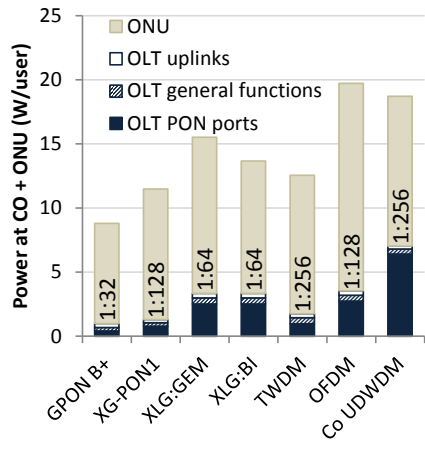

(b) optimized split
Figure 4: Power consumption at CO (OLT) and customer premises (ONU) for a reference scenario $\left(B_{\text {target }}=600 \mathrm{Mb} / \mathrm{s}\right)$. Split ratios are indicated inside the bars. Split ratio optimization decreases OLT power consumption for TWDM and OFDM PON the most. GPON B+ must be deployed with a lowered split ratio to meet QoS requirements. Overall, XG-PON1 is the most energy efficient technology that can be deployed with a split ratio $\geq 1: 64$.

units passed by fiber $(60 \%)$ and the percentage of those real estate units passed that are connected, referred to as takerate (we take $50 \%$ as a reference, and vary this value between $10 \%$ and $100 \%$ in our sensitivity analysis).

\section{Results for a reference scenario}

All power consumption calculations were implemented in Matlab; except for the city deployment model, which was implemented in Visual Basic (Excel). In our analysis of the results, we focus on the power consumption per user to compare different cases. Each case consists of a PON technology, a split ratio strategy (fixed or optimized, cf. Section 4.2), and a specific combination of the service profile parameters listed in Appendix A, quantifying user demands (numbers of users, user activity and bandwidth requirements per user) and QoS requirements (availability, packet loss). It must be noted that when the deployment has been optimized for a specific case, the switchover to another case with higher demands or stricter QoS requirements could mandate expensive changes in the ODN (e.g. lowering the split ratio). Therefore, when choosing the input parameters for our model, the cases are defined including a sufficiently large buffer to enable future growth.

This section will discuss the results for two reference cases: the fixed split and optimized split version of an average user demand scenario. The results for other cases will be discussed in the sensitivity analysis. All results include power conversion inefficiencies and site overhead where applicable (cf. equation 23).

\subsection{Total power consumption}

The stacked bars in Fig. 4 indicate the joint power consumption at the $\mathrm{CO}$ and ONU for an average user demand scenario $(600 \mathrm{Mb} / \mathrm{s})$. The values range from about $8 \mathrm{~W} / \mathrm{user}$ for GPON to more than $20 \mathrm{~W} /$ user for OFDM PON. In general, the ONU is the most important contributor (for a discussion of the technology-dependent ONU power consumption values 
we refer to Section 3.3). The share of the $\mathrm{CO}$ becomes even smaller when the split ratio is optimized (Fig. 4b, split ratios indicated inside the bars), due to a more optimal use of the OLT equipment. OFDM has the highest overall power consumption, because of the heavy digital signal processing in the ONU. Co UDWDM has the second highest total power consumption, partly due to the need for an increased number of transceivers at the $\mathrm{CO}$, but this also has a number of advantages, which will be discussed below in the detailed evaluation of the $\mathrm{CO}$ power consumption.

Which technology is the most energy efficient option overall - For the user demands in the reference scenario, GPON B+ is the most energy efficient solution, but in case of a fixed split ratio (Fig. 4a), its capacity does not suffice to serve users in an existing ODN: the probability that an active user gets the requested bandwidth drops to $60 \%$, which is below the QoS requirement of $90 \%\left(p_{\text {avail }}<p_{a v, \text { min }}\right)$. GPON B+could be deployed with a split ratio 1:32 to meet the requirements, but this would not be an attractive option for network operators, due to the increased costs associated to a higher number of OLT ports and an ODN with a higher number of fibers and ducts. In practice, XG-PON1 would therefore be a more attractive option for the near future (depending on the split ratio flexibility, it can be split 1:64 or 1:128). TWDM is an interesting option for network operators who want to increase split ratios further to achieve node consolidation, in a network that is future-proof for demands up to and beyond $1 \mathrm{~Gb} / \mathrm{s}$ (see also sensitivity analysis in the next section).

\subsection{OLT power consumption}

The lower three parts of the bars in Fig. 4 show the power consumption of the OLT PON ports, general OLT functions and OLT uplinks at the CO. Note that this power consumption is charged to the network provider.

Breakdown of the OLT contributions - The power consumption at the $\mathrm{CO}$ is mostly dominated by the contribution of the PON ports, while power consumption of the general OLT functions and uplinks is much lower. For GPON B+ however, general functions and uplinks are the dominant contributors, due to its relatively low power per OLT PON port (see also Table 1). Similarly, in case of TWDM with an optimized split ratio, where the power/user for PON ports is low due to a large number of users sharing each port, the contribution of general OLT functions and uplinks is relatively important. As user demands increase (not shown in the graph), the contribution of the general OLT functions and uplinks will grow for all technologies, since they scale with the capacity and traffic load. If the bandwidth per user $B_{\text {target }}$ goes up to $1 \mathrm{~Gb} / \mathrm{s}$, general OLT functions and uplinks for NG-PON2 technologies will consume about $1.3 \mathrm{~W} /$ user in a fixed split scenario, and about $1 \mathrm{~W} / \mathrm{user}$ in an optimized split scenario.

Impact of split ratio optimization - When the split ratio is fixed, NG-PON2 technologies have a much higher energy demand at the $\mathrm{CO}$ compared to the existing technologies, due to the high line rates. Evidently, sharing a $40 \mathrm{~Gb} / \mathrm{s}$ PON capacity among 32 connected users (split ratio 1:64, takerate 50\%) is a substantial overprovisioning, which comes at a high energy cost. Optimizing the split ratio reduces power consumption at the CO for TWDM and OFDM PON the most. Thanks to their high optical budgets and capacities, many users can be connected to a single OLT port, thus equipment sharing can make these technologies more energy efficient. XLG PON ${ }^{8}$ has a lower optical budget, so even though it offers the same per-PON capacity as TWDM and OFDM, its maximum split ratio is 1:64, resulting in a high energy consumption per subscriber regardless of split ratio flexibility. Co UDWDM is clearly the most power-hungry technology in every scenario. However, it must be noted that this solution offers the advantage of $100 \%$ bandwidth availability on the first mile and lowest traffic latency, which may be useful for specific applications such as business services or mobile backhauling and fronthauling. Moreover, the link budget is very high, and user demands can always be met thanks to the dedicated wavelength channels, therefore this technology can always be deployed with split ratio 1:256 (or even higher), even for very high user demands.

Which technology is the most energy sparing for network operators - As mentioned before, though GPON B+ has the lowest power consumption, it does not have sufficient capacity to offer user rates up to $600 \mathrm{Mb} / \mathrm{s}$ with $90 \%$ availability when the split ratio is 1:64. Among the other options, XG-PON1 is not only the most energy efficient solution overall, but also the most energy efficient solution at the $\mathrm{CO}$ for user demands up to $600 \mathrm{Mb} / \mathrm{s}$.

Effects of statistical multiplexing - It is interesting to note that for XG-PON1, even though the total PON bandwidth divided by the number of connected users is below the target bandwidth, statistical multiplexing allows for an availability of more than $90 \%$, since not all users are active at the same time. The same is true for TWDM PON in case of an optimized split ratio. As demands increase, XLG and OFDM PON could also take advantage of this statistical multiplexing effect, but in this reference scenario, their split ratios are limited by reach restrictions, so their capacity is not fully exploited.

\section{Impact of parameter variations on results (sensitivity analysis)}

Since most of our input parameters are estimates based on literature rather than experimentally verified values, they come with some uncertainty. In order to know the impact of this uncertainty on our results, we perform a sensitivity analysis. We vary fourteen input parameters along the distributions given in Appendix A, and study the variations in the $\mathrm{CO}$ power consumption as a result of these input variations. We only focus on power consumption at the $\mathrm{CO}$, since the ONU power consumption is assumed constant and therefore a sensitivity analysis for the ONU is straightforward: if there is $10 \%$ deviation in the input for ONU power consumption, this results in $10 \%$ deviation in the ONU result. The results we show do however include ONU power consumption, as we want to compare the overall energy efficiency between technologies.

\footnotetext{
${ }^{8}$ We do not distinguish between XLG:GEM and XLG:BI on the OLT side, as they require identical OLT equipment.
} 


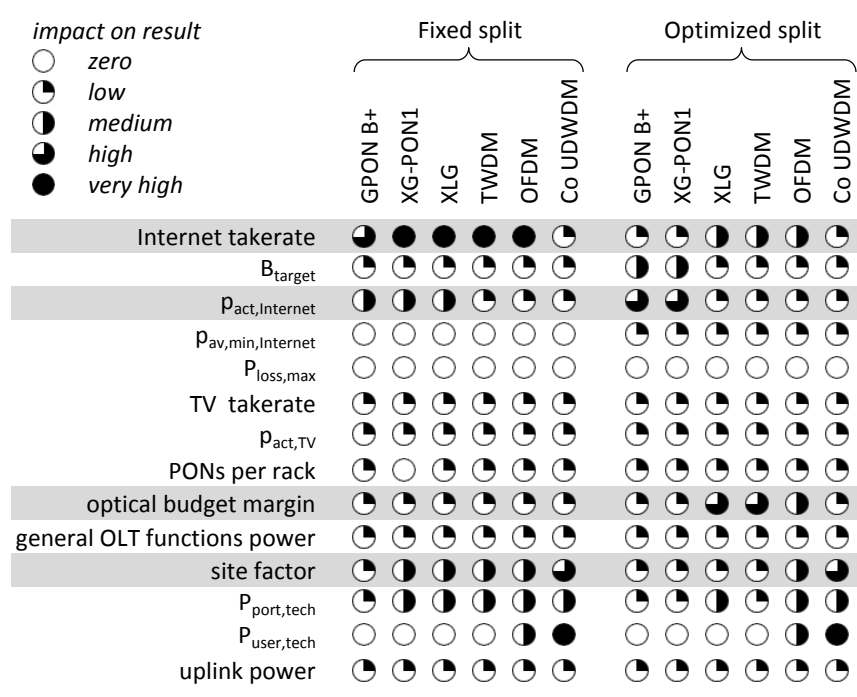

Figure 5: Relative importance of the input parameter variations for each technology. The effect of varying some of the most impacting parameters (highlighted in gray) is shown in Fig. 6.

For each of the fourteen varying input parameters, we calculate the first-order sensitivity index per technology for both fixed and optimized split ratios. The first-order sensitivity index [26] quantifies the contribution of a given input parameter to the variance of the output - in this case, the $\mathrm{CO}$ power consumption. A qualitative indication of the sensitivity indices is presented in Fig. 5. Note that icons are to be compared within columns, since the sensitivity analysis is performed separately for each technology. As an example of how to interpret the symbols in Fig. 5, consider GPON with a fixed split ratio. The three-quarter-full disc in the first row indicates that most of the variation in the power consumption for GPON comes from variations in Internet takerate. The half-full disc for active user probability indicates that this parameter variation also causes significant output changes. The impact of the other parameter variations is relatively minor for GPON with a fixed split ratio.

The four most impacting parameters overall are highlighted in gray in Fig. 5. We will discuss the effect of varying each of these parameters individually. But first, we will look into the effect of varying the bandwidth per user between $100 \mathrm{Mb} / \mathrm{s}$ (current demands) and $1 \mathrm{~Gb} / \mathrm{s}$ (future demands).

\subsection{Impact of varying bandwidth per user}

For current user demands $\left(B_{\text {target }}=100 \mathrm{Mb} / \mathrm{s}\right), \mathrm{GPON} \mathrm{B}+$ is by far the most energy efficient option, both for fixed and optimized split ratios. If future users require target bandwidths up to $1 \mathrm{~Gb} / \mathrm{s}$ and the split ratio is fixed (not shown in graphs), the effect on power per user is similar for all technologies: an increase of about $0.5 \mathrm{~W} /$ user compared to the $600 \mathrm{Mb} / \mathrm{s}$ reference scenario. Consequently, XG-PON1 remains the most energy efficient technology for future demands up to $1 \mathrm{~Gb} / \mathrm{s}$ in case of a fixed split ratio. When the split ratio is optimized, the added degree of freedom leads to a less predictable effect of the target bandwidth changes, shown in Fig. 6a. For target bandwidths up to $900 \mathrm{Mb} / \mathrm{s}, \mathrm{XG}-\mathrm{PON} 1$ can be deployed with a split ratio of
1:128, consuming less power per user than TWDM PON. For demands going up to $1 \mathrm{~Gb} / \mathrm{s}$ however, the capacity limitation of XG-PON1 mandates a split ratio reduction to 1:64, bringing its energy efficiency at the same level as that of TWDM PON. The latter might be a more attractive option in this case, due to its higher split ratio (1:256) and potential for node consolidation.

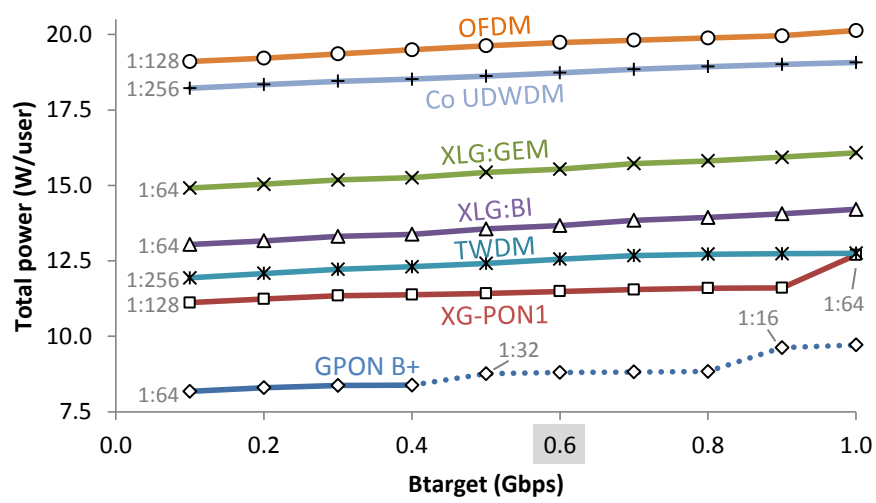

(a) For user rates up to $1 \mathrm{~Gb} / \mathrm{s}$, TWDM reaches the same energy efficiency as XG-PON1.

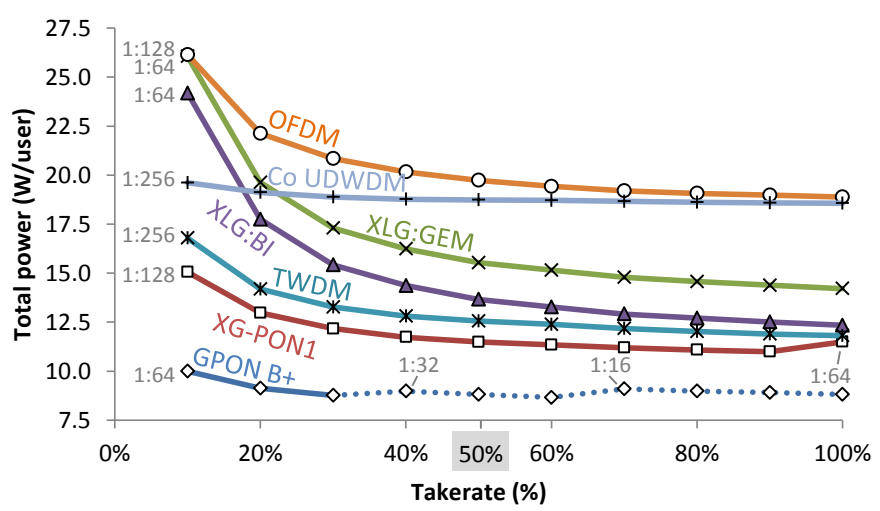

(b) Low takerates worsen energy efficiency, except for Co UDWDM.

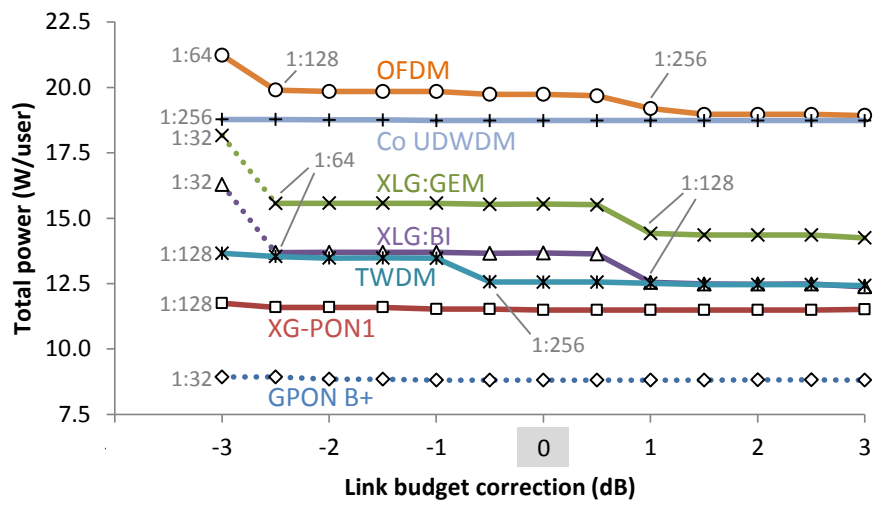

(c) With an improved link budget, XLG:BI reaches the same energy efficiency as TWDM.

Figure 6: Sensitivity of the result (total power $=\mathrm{CO}+\mathrm{ONU}$ ) to changes in the most impacting input parameters, in case of optimized split ratios. The shaded value on the horizontal axis is that of the reference scenario. Split ratio changes are indicated in gray. Dotted lines indicate data points with split ratios $<1: 64$, these cases would result in unacceptable QoS in a fixed split ratio scenario. The results for varying active user probability are not shown, as they show the same trends as for varying $B_{\text {target }}$. 


\subsection{Impact of varying other input parameters}

The Internet takerate is the most impacting input parameter for all technologies in case of fixed split ratios, because as the takerate increases, the number of users sharing PON equipment increases, resulting in a lower power per user. Takerates below $30 \%$ result in much higher power per user compared to the reference scenario with $50 \%$ takerate (e.g. for $30 \%$ takerate, TWDM power per user is $2.6 \mathrm{~W}$ higher than for the reference case). For optimized split ratios (Fig. 6b), the effect is less pronounced, but still significant. For takerates below $20 \%$, there is a notable deterioration in energy efficiency for all technologies. Note that the impact for Co UDWDM is limited for both fixed and optimized split ratios, since the installed capacity for this technology scales better with the number of connected users.

The results for varying active user probability show similar trends as for varying $B_{\text {target }}$ (cf. Section 7.1), as they ultimately have the same effect: an increase in the PON traffic load and in the required OLT capacity.

The site factor is applied to all power consumption values at the $\mathrm{CO}$, so it directly impacts power consumption: varying the site factor with $10 \%$ scales the total $\mathrm{CO}$ power consumption with $10 \%$, this is the case for both fixed and optimized split ratio scenarios.

Variations in the optical budget margin generally have a limited effect when the split ratio is fixed (not shown in graphs), though a shorter range could result in slightly less equipment sharing due to less centralized COs. In case of optimized split ratios, the optical budget margin is an important parameter for XLG PON, TWDM PON and OFDM PON, as it can be a limiting factor for the split ratio optimization. Due to their high capacities, reach is typically the limiting factor for these technologies. Figure $6 \mathrm{c}$ shows that XLG:BI could achieve the same power/user if the link budget was improved by $1 \mathrm{~dB}$ (for example by making the margin for fiber patching more strict). But in any case, TWDM keeps the advantage of the highest split ratio potential.

\subsection{Conclusion of the sensitivity analysis}

Though changes in the input parameters can result in significant variations in the $\mathrm{CO}$ power consumption for individual technologies, the relative proportions between the technologies are preserved in most cases, so the comparison between the technologies still holds. Although our sensitivity analysis focused mainly on the $\mathrm{CO}$, note that changes in the ONU will most likely have a bigger impact, due to its bigger contribution to the overall power per user.

\section{Conclusion}

We studied the power consumption of various nextgeneration PON technologies for a massive residential deployment. The choice for a fixed split ratio or optimized split ratio strategy will impact which technology is the most energy efficient option for future deployments with access bandwidths up to $1 \mathrm{~Gb} / \mathrm{s}$ : XG-PON1 (with split ratio 1:64) or TWDM PON (with split ratio 1:256). TWDM PON can take advantage of its high optical power budget, resulting in a long reach that allows node consolidation (reducing the number of central offices and OLT ports), which can help reduce both capital expenditures and operational costs for network operators.

We also analyzed the sensitivity of our results to the input parameters of our model. As it turns out, variations in takerate, active user probability, optical budget margin and site factor have the biggest impact on our result for the power consumption at the central office. Reducing the site factor, for example by using energy-efficient cooling systems, can significantly reduce power consumption. Further, a high takerate significantly reduces power consumption per user due to improved equipment sharing, which brings the actual traffic load closer to the provisioned capacity. This shows that a scenario where multiple operators each have their own optical access network, is not desirable from an energy efficiency point of view. An open access scenario, where multiple operators share the passive optical network infrastructure [27], could be a more energy efficient solution from network operators' perspective. But the biggest impact on the overall power consumption can be made by improving the energy efficiency of ONUs, as they still consume the bulk of optical access network energy. The inclusion of energy saving strategies such as sleep modes or energy efficient protocols in the comparison can have a big impact on the results, as illustrated by the improved energy efficiency of XLG:BI compared to XLG:GEM.

To conclude, we would like to remark that in the current work, our focus has been on energy efficiency with current technology maturity and system performance estimations, and without taking into account cost aspects. TWDM PON, which was selected by FSAN as the main solution for NG-PON2 for its lower expected cost than other candidates and commercial viability by 2015 , also turns out to be an energy efficient solution for residential services. For other services with specific requirements, other characteristics (such as system capacity, bandwidth availability and latency) may be prioritized over energy efficiency or cost.

\section{Appendix A. Input parameters for the sensitivity analysis}

Table A. 3 contains an overview of the input parameters that are varied between simulations in the sensitivity analysis. The first column shows the default values of the reference scenario. The other columns show the restrictions for the random variation of the parameters in the sensitivity analysis. The distribution of the parameters for the sensitivity analysis is Gaussian, except when specified otherwise.

\section{Acknowledgment}

This research has received funding from the EU FP7 projects TREND (ICT-257740) and DISCUS (ICT-318137). The first author is funded by the Agency for Innovation by Science and Technology in Flanders (IWT). Part of this research was performed in the context of GreenTouch. 
Table A.3: Overview of input parameter variations for the sensitivity analysis.

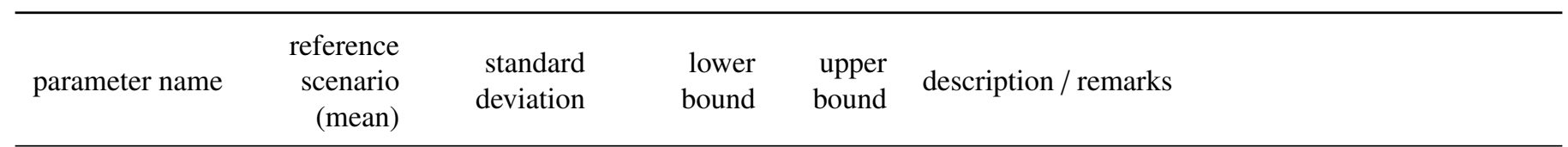

\section{User demand: Internet service profile}

Internet takerate

$50 \%$

$10 \%$

$B_{\text {target }}$

$$
600 \mathrm{Mb} / \mathrm{s}
$$

$200 \mathrm{Mb} / \mathrm{s}$

pact,Internet

$\mathrm{p}_{\mathrm{av}, \mathrm{min}, \text { Internet }}$

$10 \%$

$5 \%$

$90 \%$

$10 \%$

$\log _{10}\left(\mathrm{P}_{\text {loss,max }}\right)$

User demand: TV service profile

TV takerate

$25 \%$

$10 \%$

$\mathrm{p}_{\text {act,TV }}$

$60 \%$

$10 \%$

Physical characteristics of technologies

PONs per rack

cf. Table 1

$3 \mathrm{~dB}$

$1.5 \mathrm{~dB}$

$L_{m}$

\section{Power consumption calculation}

\section{general OLT}

functions power

$1 \mathrm{~W} / \mathrm{Gbps} \quad 0.1 \mathrm{~W} / \mathrm{Gbps}$

$0 \mathrm{~W} / \mathrm{Gbps}$

site factor

1.6

0.16

1

$\mathrm{P}_{\text {port,tech }}$

cf. Table 1

$10 \%$ of

$\mathrm{P}_{\text {user,tech }}$

cf. Table 1

mean

$10 \%$ of

uplink Ethernet

ports power cf. page 7 mean

$10 \%$ of mean
Percentage of homes passed by fiber that are connected.

The sustainable bandwidth requested by users when they are active.

Probability that a user is active, requesting $\mathrm{B}_{\text {target }}$.

QoS parameter: minimum probability that a user will get $\mathrm{B}_{\text {target }}$ when requested.

QoS parameter: exponent for maximum allowable

-3 packet loss in the uplink. Distribution is flat, not Gaussian.

$0 \% \quad 100 \% \quad$ Percentage of Internet subscribers that have IPTV.

$0 \% \quad 100 \% \quad$ Percentage of TV sets switched on during peak hour.

$50 \%$ of $2 \times \quad$ Number of PONs per rack, rounded to the nearest mean mean integer.

Margin for fiber patching, subtracted from the (none) optical link budget to calculate reach, see equation (7).

Power consumption factor for switching, traffic (none) management and packet processing. Unidirectional, excluding overhead factors.

2 CO power consumption is multiplied by the site factor (defined in Section 2).

OLT power per port for opto-electronic components, excluding overhead factors.

OLT power per user for opto-electronic components, excluding overhead factors.

Power consumption of the uplink Ethernet ports from [24]. 
We would like to thank L. Guan and M. Ruffini from The Telecommunications Research Center (CTVR), Dublin, Ireland, for providing the IPTV channel popularity information.

\section{References}

[1] P. Chanclou, A. Cui, F. Geilhardt, H. Nakamura, D. Nesset, Network operator requirements for the next generation of optical access networks, Network, IEEE 26 (2) (2012) 8-14. doi : 10.1109/MNET . 2012.6172269.

[2] B. Skubic, E. De Betou, T. Ayhan, S. Dahlfort, Energy-efficient nextgeneration optical access networks, Communications Magazine, IEEE 50 (1) (2012) 122-127. doi:10.1109/MCOM . 2012.6122542.

[3] K. Grobe, M. Roppelt, A. Autenrieth, J.-P. Elbers, M. Eiselt, Cost and energy consumption analysis of advanced WDM-PONs, Communications Magazine, IEEE 49 (2) (2011) s25-s32. doi:10.1109/MCOM. 2011. 5706310.

[4] D. Suvakovic, H. Chow, D. van Veen, J. Galaro, B. Farah, N. Anthapadmanabhan, P. Vetter, A. Dupas, R. Boislaigue, Low energy bitinterleaving downstream protocol for passive optical networks, in: IEEE Online Conference on Green Communications (GreenCom), 2012, pp. 26-31. doi:10.1109/GreenCom.2012.6519611.

[5] J. Montalvo, J. Torrijos, J. Xia, Y. Ye, Energy efficiency in PON home network scenarios with network enhanced residential gateways, in: 10th IEEE International Conference on Networking, Sensing and Control (ICNSC), 2013, pp. 141-145. doi : 10.1109/ICNSC. 2013.6548726.

[6] F. Saliou, P. Chanclou, N. Genay, F. Laurent, F. Bourgart, B. Charbonnier, Energy efficiency scenarios for long reach PON central offices, in: Optical Fiber Communication Conference/National Fiber Optic Engineers Conference (OFC/NFOEC), Optical Society of America, 2011, p. OThB2. doi:10.1364/OFC. 2011. OThB2.

[7] S. Lambert, J. Montalvo, J. Torrijos, B. Lannoo, D. Colle, M. Pickavet, Energy demand of high-speed connectivity services in NG-PON massive deployments, in: 39th European Conference and Exhibition on Optical Communication (ECOC), 2013, p. P.6.5. doi:10.1049/cp. 2013. 1661.

[8] S. Lambert, J. Montalvo, J. A. Torrijos, B. Lannoo, D. Colle, M. Pickavet, Energy efficiency analysis of next-generation passive optical network (NG-PON) technologies in a major city network, in: 15th International Conference on Transparent Optical Networks (ICTON), 2013. doi : 10.1109/ICTON.2013.6602846.

[9] OASE, Integrated OASE results overview (D8.5) (March 2013). URL http://www.ict-oase.eu/public/files/OASE_D8.5_WP8_ DTAG_15032013_V1.0.pdf

[10] H. Chow, D. Suvakovic, D. van Veen, A. Dupas, R. Boislaigue, R. Farah, M. F. Lau, J. Galaro, G. Qua, N. P. Anthapadmanabhan, G. Torfs, C. V. Praet, X. Yin, P. Vetter, Demonstration of low-power bit-interleaving TDM PON, in: 38th European Conference and Exhibition on Optical Communication (ECOC), Optical Society of America, 2012, p. Mo.2.B.1. doi:10.1364/ECEOC. 2012.Mo.2.B.1.

[11] S. Smolorz, H. Rohde, E. Gottwald, D. W. Smith, A. Poustie, Demonstration of a Coherent UDWDM-PON with real-time processing, in: Optical Fiber Communication Conference/National Fiber Optic Engineers Conference (OFC/NFOEC), Optical Society of America, 2011, p. PDPD4.

[12] A. Dixit, S. Lambert, B. Lannoo, D. Colle, M. Pickavet, P. Demeester, Towards energy efficiency in optical access networks, in: IEEE International Conference on Advanced Networks and Telecommunications Systems (ANTS), 2013. doi : 10.1109/ANTS . 2013.6802896.

[13] A. Dixit, B. Lannoo, D. Colle, M. Pickavet, P. Demeester, ONU power saving modes in next generation optical access networks: progress, efficiency and challenges, Optics Express 20 (26) (2012) B52-B63. doi : 10.1364/OE. 20.000B52.

[14] Ofcom, Communications market report 2013 (August 2013). URL http: //stakeholders . of com.org.uk/

[15] A. Ali, S. Vassilaras, K. Ntagkounakis, A comparative study of bandwidth requirements of VoIP codecs over WiMAX access networks, in: Third International Conference on Next Generation Mobile Applications, Services and Technologies (NGMAST), 2009, pp. 197-203. doi : 10.1109/NGMAST . 2009.47.

[16] Analysys Mason, The connected consumer survey 2013: TV and video (May 2013).
URL http://www. analysysmason.com/Research/Content/ Reports/Connected-Consumer-TV-May2013-RDMB0/

[17] D. T. v. Veen, M. K. Weldon, C. C. Bahr, E. E. Harstead, An analysis of the technical and economic essentials for providing video over fiber-tothe-premises networks, Bell Labs Technical Journal 10 (1) (2005) 181200. doi:10.1002/bltj. 20088.

[18] D. E. Smith, IP TV Bandwidth Demand: Multicast and Channel Surfing, in: 26th IEEE International Conference on Computer Communications (INFOCOM), 2007, pp. 2546-2550. doi : 10.1109/INFCOM. 2007.318.

[19] J. Segarra, V. Sales, J. Prat, Access services availability and traffic forecast in PON deployment, in: 13th International Conference on Transparent Optical Networks (ICTON), 2011, pp. 1-6. doi : 10.1109/ICTON. 2011.5970909.

[20] E. Goma, M. Canini, A. Lopez Toledo, N. Laoutaris, D. Kostić, P. Rodriguez, R. Stanojević, P. Yagüe Valentin, Insomnia in the access: or how to curb access network related energy consumption, in: Special Interest Group on Data Communication Conference (SIGCOMM), ACM, New York, USA, 2011, pp. 338-349. doi : 10.1145/2018436. 2018475.

[21] OECD, OECD Communications Outlook 2013, ISBN 978-92-64-194595.

[22] European Commission, Quality of Broadband Services in the EU (March 2012).

URL http://ec.europa.eu/digital-agenda/en/news/ quality-broadband-services-eu-march-2012

[23] W. Lautenschlager, W. Frohberg, Bandwidth dimensioning in packetbased aggregation networks, in: The 13th International Telecommunications Network Strategy and Planning Symposium (Networks), 2008, pp. 1-8. doi:10.1109/NETWKS.2008.4763737.

[24] W. Van Heddeghem, F. Idzikowski, W. Vereecken, D. Colle, M. Pickavet, P. Demeester, Power consumption modeling in optical multilayer networks, Photonic Network Communications 24 (2) (2012) 86-102. doi: 10.1007/s11107-011-0370-7.

[25] Spanish National Institute of Statistics, Population of the continuous municipal register by population unit, http: //www. ine.es.

[26] A. Saltelli, M. Ratto, T. Andres, F. Campolongo, J. Cariboni, D. Gatelli, M. Saisana, S. Tarantola, Variance-Based Methods, John Wiley \& Sons, Ltd, 2008, pp. 155-182. doi : 10.1002/9780470725184.ch4.

[27] A. Banerjee, G. Kramer, B. Mukherjee, Fair sharing using dual servicelevel agreements to achieve open access in a passive optical network, Selected Areas in Communications, IEEE Journal on 24 (8) (2006) 32-44. doi : 10.1109/JSAC. 2006.1677253.

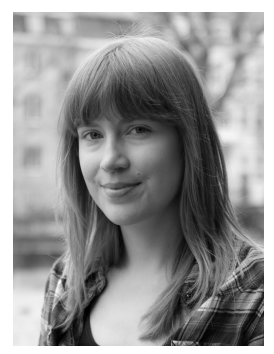

Sofie Lambert is a Ph.D. candidate at Ghent University (Belgium). She received her M.Sc. degree in Photonics from Ghent University in 2011, and joined the Green ICT research team of the Information Technology department (INTEC) of Ghent University in 2012. She studies the electricity consumption of ICT infrastructures, specifically in communication networks. Her current research is focused on the energy efficiency of future access network architectures, both optical and wireless, and the impact of energy saving strategies such as sleep modes on these networks. 


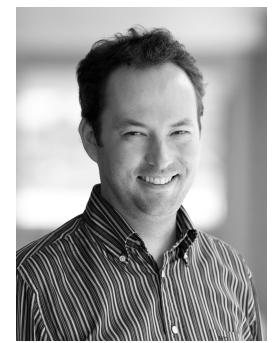

Bart Lannoo received a M.Sc. degree in Electrotechnical engineering and a Ph.D. degree from Ghent University (Belgium) in July 2002 and May 2008, respectively. Since August 2002, he has been working at the Internet Based Communication Networks and Services (IBCN) research group of the Department of Information Technology (INTEC) of Ghent University. He is also affiliated with the research institute iMinds. His main research interests are fixed and wireless access networks, and he coordinates the GreenICT research at IBCN. He is author or co-author of more than 100 international publications, both in journals and in proceedings of conferences.

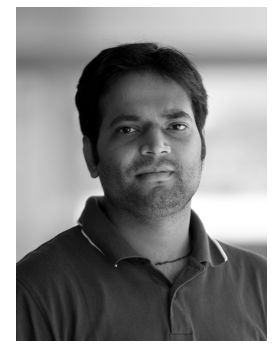

Abhishek Dixit received the M.Tech. degree in Optical Electronics and Optical Communication from the Indian Institute of Technology, Delhi (India) in 2010. He is currently working towards the Ph.D. degree from Ghent University, Belgium. He was active in the European project ICTOASE and has more than 20 scientific publications in international conferences and journals. His research interests include broadband optical access networks and Internet of things (IoT).

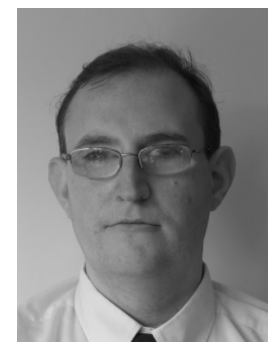

Didier Colle received a M. Sc. degree in Electrotechnical engineering (option: communications) from Ghent University in 1997. Since then, he has been working at the same university as researcher in the department of Information Technology (INTEC). He is part of the research group Internet Based Communication Networks and Services (IBCN) headed by prof. Piet Demeester. His research lead to a Ph.D. degree in February 2002. He became professor at Ghent University in October 2011. Currently, he is co-responsible for the research cluster on network modelling, design and evaluation and is coordinating the research on fixed internet architectures and optical networks.

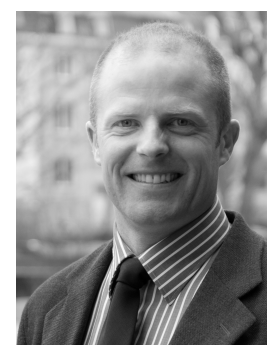

Mario Pickavet is full professor at Ghent University where he is teaching courses on discrete mathematics, broadband networks and network modeling. He is leading research tracks on fixed internet architectures and optical networks, green ICT and design of network algorithms. He has published about 300 international publications, both in journals (IEEE JSAC, IEEE Comm. Mag., Journal of Lightwave Technology, Proceedings of the IEEE, ...) and in proceedings of conferences. He is coauthor of the book 'Network Recovery: Protection and Restora- tion of Optical, SONET-SDH, IP, and MPLS'.

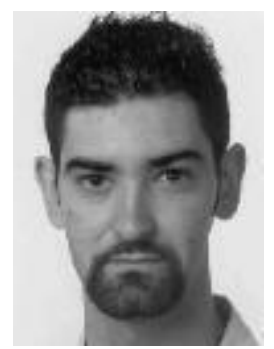

Julio Montalvo holds an M.Sc. degree in telecommunication engineering (2003) from the Technical University of Madrid and a Ph.D. degree in electrical, electronics and robotics engineering (2008) from Carlos III University of Madrid. He is the author of more than 30 papers in research journals and conferences, one book chapter and one granted patent. He has been involved in several European research projects. He joined Telefónica I+D in 2011 as project manager in fixed access and home networks. He is also Telefónicas delegate in the Broadband Forum and Full Service Access Networks.

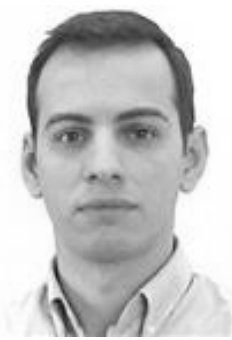

Jose Alfonso Torrijos Gijón has a Computer Science degree from the Polytechnic University of Madrid in 1992. In 1994 he joined Telefnica I+D working in different projects for Telefónica regarding IP, DTV, xDSL and Satellite technologies. In 2004 he joined the Broadband Access Technologies Division managing ARTES-1 and FP6 projects such as SATLIFE and SATSIX. In 2008 he started to work in fibre optic projects for Telefónica Spain regarding FTTH access and photonic networks where he is currently involved.

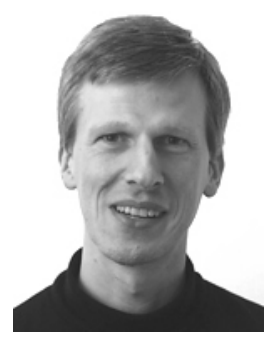

Peter Vetter is Head of the Fixed Networks Program in Bell Labs. He is globally responsible for research on copper and optical access, access hardware platforms, and access architectures. He received the degree of Physics Engineer from Ghent University (Belgium) in 1986 and a Ph.D. in 1991. After a post-doctoral fellowship at Tohoku University (Japan), he joined the research center of Alcatel (now Alcatel-Lucent) in Antwerp, Belgium, in 1993. Since 2009, he is with Bell Labs in Murray Hill, New Jersey. During his career, he subsequently worked on liquid crystal displays, optical interconnections, optical access, access platforms, access network architectures, and green access networks. 